\title{
Políticas y Negocios para la Bioeconomía en ALC: Un proceso en marcha
}

\author{
Hugo Chavarría ${ }^{1}$, Eduardo Trigo ${ }^{1}$ y Juan F. Martínez ${ }^{1}$
}

Autor de Correspondencia: hugo.chavarria@iica.int

\section{Resumen:}

A nivel productivo, desde hace más de 30 años diversos países de América Latina y el Caribe gestan modelos de negocios que transitan por diferentes vías de la bioeconomía. Hoy algunos de esos países son líderes en aplicaciones biotecnológicas para la agricultura, bioenergías, aprovechamiento de la biodiversidad y agricultura baja en carbono. En el ámbito políticoinstitucional, el concepto de bioeconomía llegó a la región de la mano de proyectos impulsados por la cooperación europea, que permitieron avanzar en la sensibilización del tema. En paralelo, los países han desarrollado iniciativas y normativas para aquellos senderos de la bioeconomía de mayor potencial. Solo recientemente los países iniciaron la construcción de políticas y estrategias dedicadas específicamente a la bioeconomía. Además de los esfuerzos nacionales, la cooperación internacional ha desempeñado un papel importante en la promoción de iniciativas regionales para la construcción de bienes públicos y el aprovechamiento de buenas prácticas y lecciones aprendidas. Si bien en la región se han logrado avances importantes, el aprovechamiento de la bioeconomía requiere mayores esfuerzos destinados a la construcción de institucionalidad, políticas e instrumentos de mercados que no solo viabilicen negocios rentables, sino que también aseguren un marco de seguridad y sostenibilidad.

Palabras clave: América Latina y el Caribe, biotecnología, biocombustibles, bionegocios, biodiversidad

\section{Policies and Business for the Bioeconomy in LAC: An ongoing process}

\author{
Hugo Chavarría ${ }^{1}$, Eduardo Trigo ${ }^{1}$ y Juan F. Martínez ${ }^{1}$
}

\begin{abstract}
:
At the productive level, for more than 30 years, several countries in Latin America and the Caribbean have developed business models related to different bioeconomy pathways. Today some of these countries are leaders in biotechnological applications for agriculture, bioenergies, use of biodiversity and low carbon agriculture. In the political-institutional sphere, the concept of bioeconomy was established in the region through projects assisted by the European cooperation, which promoted the awareness of this topic. In parallel, the countries have elaborated initiatives and regulations for those bioeconomy paths with the greatest potential. Just recently countries started to build policies and strategies specifically dedicated to the bioeconomy. In addition to national efforts, international cooperation has played an important role in promoting regional initiatives for the construction of public goods and the use of good practices and lessons learned. Although important progress has been made in the region, the exploitation of bioeconomy requires greater efforts focused on institution building, policies and market instruments that not only make profitable businesses viable but also ensure a framework of security and sustainability.
\end{abstract}

Key Words: Latin America and the Caribbean, biotechnology, biofuels, bio-businesses, biodiversity

I Instituto Interamericano de Cooperación para la Agricultura (IICA), (Costa Rica), hugo.chavarria@iica.int, ejtrigo@gmail.com, ifmartinez17@gmail.com

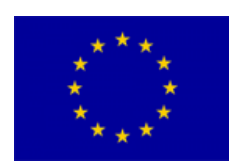

UNIÓN EUROPEA

PROYECTO COFINANCIADO POR EL FONDO EUROPEO DE DESARROLLO REGIONAL (FEDER)

Una manera de hacer Europa

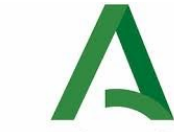

Junta de Andalucía
Andalucía

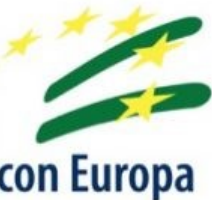




\section{El ATERRIZAJE DE LA BIOECONOMÍA EN ALC}

En términos generales, se podría decir que la aplicación de la bioeconomía se inició en América Latina y el Caribe (ALC) a comienzos de la década de 1970 con la instalación del programa "Pró-álcool" en Brasil, como respuesta al aumento del precio internacional del petróleo, que dio origen a la creación de la Organización de Países Exportadores de Petróleo (OPEP). Más tarde, en los años noventa, este proceso tuvo un importante impulso con los desarrollos vinculados a las biotecnologías emergentes, especialmente en la creación de capacidades y marcos regulatorios, que ocasionó la rápida adopción en algunos países -en particular, Argentina- de los primeros cultivos genéticamente modificados (GM) y las prácticas de agricultura conservacionista. En consecuencia, la región se transformó en líder mundial en la implementación de este tipo de estrategias productivas (Trigo et al., 2009a, 2009b).

La idea de la bioeconomía como estrategia para el desarrollo sostenible aparece tiempo después vinculada a iniciativas de la cooperación internacional. Distintos proyectos birregionales entre la Unión Europea (UE) y ALC permitieron la instalación del debate de la bioeconomía como visión para el desarrollo sostenible en la región, en el ámbito de los organismos públicos responsables de las políticas de ciencia, tecnología e innovación (CTI). Por ejemplo, en el marco del proyecto ALCUE-FOOD (European Commission, 2008) del Programa IV de la UE, en 2008 se organizó un taller regional en Buenos Aires, Argentina. A partir de este, surgió el compromiso institucional de llevar adelante un esfuerzo colaborativo entre los países de ALC y la UE interesados en el tema, para promover el desarrollo de una visión compartida de la bioeconomía, además de una perspectiva particular y diferenciadora (Trigo \& Henry, 2011).

Paralelamente, la Organización de las Naciones Unidas para el Desarrollo Industrial (ONUDI) propuso, entre 2008 y 2009, la integración de un grupo de trabajo sobre el tema. Este grupo de expertos, provenientes de más de diez países de ALC y la UE, entregaron un informe que resaltó las oportunidades de la bioeconomía para la región y una serie de recomendaciones para la acción. 
Dentro de estas se destacaron el diseño de políticas y toma de decisiones, la construcción de capacidades y la facilitación del desarrollo empresarial en el sector (UNIDO, 2009).

En este contexto de interés en la bioeconomía, se aprobó en el marco del Programa VII de la UE, el proyecto ALCUE-KBBE "Hacia una bioeconomía basada en el conocimiento de América Latina y el Caribe en asociación con Europa". Su objetivo fue establecer una plataforma cooperativa ALC-UE para sentar las bases de un entorno político e institucional propicio para el desarrollo de la bioeconomía. Este proyecto contó con la participación de once países (Francia, Alemania, Países Bajos, Bélgica, Portugal, Argentina, Colombia, Costa Rica, Brasil, Uruguay, México) y se llevó a cabo entre 2011 y 2013 (European Commission, 2013). Se puede considerar este período como la maduración definitiva de la idea de la bioeconomía como visión para el desarrollo sostenible y la etapa de inclusión en la agenda política de la región (Hodson 2014). Esto se reflejó claramente en la implementación del proyecto ALCUE-NET ("Red de Investigación e Innovación de América Latina, el Caribe y la Unión Europea") en 2012-2017, el cual creó formalmente un espacio de bioeconomía dentro de la estructura de los diálogos políticos birregionales encargados de implementar las decisiones de las Cumbres de los Jefes de Estado de la UE-ALC (European Commission, 2017).

\section{LA ADOPCIÓN TEMPRANA Y EL PROTAGONISMO EN LOS NEGOCIOS DE LA BIOECONOMÍA: UN EFECTO DEMOSTRACIÓN QUE SIRVIÓ PARA CONVENCER A LOS TOMADORES DE DECISIÓN}

En ALC, los negocios de la bioeconomía iniciaron mucho antes que las políticas y las estrategias enfocadas en el tema. Para mediados de la década de los noventa, la región comenzó a utilizar nuevas tecnologías para aprovechar de manera más eficiente y sostenible los recursos biológicos en la agricultura y en otras ramas económicas. Aunque en aquellos momentos se le denominaba diferente, lo cierto es que diversos países de ALC fueron adoptantes tempranos 
y hoy son referentes mundiales en algunos negocios de la bioeconomía, como en la producción y exportación de biocombustibles líquidos, aplicaciones biotecnológicas en la agricultura, carbono neutralidad en las cadenas agrícolas y aprovechamiento sostenible de la biodiversidad, entre varios otros.

En bioetanol, por ejemplo, cerca de 14 países de América Latina han establecido mandatos para el uso obligatorio de la mezcla del etanol con los combustibles convencionales, que va desde el $5 \%$ en Guatemala y Uruguay hasta el $27 \%$ en Brasil (REN21, 2019). En este último, la caña de azúcar y sus derivados contribuyen con más del $17 \%$ de la energía primaria y sustituyen el 36 \% de la gasolina; de ahí que este país sea el segundo productor mundial (detrás de los Estados Unidos) y el primer exportador de etanol de caña de azúcar del mundo (Stolf \& Oliveira, 2020). Para el caso de la industria del biodiésel, Argentina es el tercer productor, segundo consumidor y primer exportador global. Además, se destaca el caso de Colombia, el único país de la región que utiliza diésel de palma para hacer frente al total de la mezcla obligatoria de biocombustibles ( $10 \%$ ) y es el líder en ALC en la producción de biodiésel de este origen (CEPAL et al. 2019).

Por otra parte, en 1996 Argentina fue pionera en la introducción y adopción de la soja tolerante a herbicidas, adelantándose a países como Australia e India (James, 1997). Hoy prácticamente la totalidad de la soja producida en Argentina (así como el maíz y el algodón) son cultivos GM (Trigo, 2016). Además de Argentina, este tipo de cultivos se ha expandido a Brasil, Paraguay, Uruguay, Bolivia, México, Colombia, Honduras, Chile y Costa Rica, cubriendo más de 82 millones de hectáreas (ISAAA, 2018). Dentro de los beneficios de la adopción de los cultivos GM se encuentra el mayor desempeño productivo y la generación de impactos favorables en materia de sostenibilidad ambiental (Brookes \& Barfoot 2018). De acuerdo con ISAAA (2018), el uso de OGM en la agricultura del mundo ha permitido conservar 183 millones de hectárea de tierra y ahorrar 671 millones de $\mathrm{kg}$ de pesticidas en los últimos diez años; y solamente en el 2016 posibilitó la reducción de emisiones de $\mathrm{CO}_{2}$ en aproximadamente 27 billones de $\mathrm{kg}$ (equivalente a 16 millones de carros rodando por año). En la actualidad, la región ha desarrollado sus propios cultivos GM, tales como el frijol resistente al virus del mosaico dorado (Aragão, 2009) y la 
soja y el trigo tolerantes a la sequía (Waltz, 2015), y avanza en el uso de nuevas tecnologías de mejoramiento genético; por ejemplo, en Colombia fueron aprobadas variedades de arroz resistente al tizón bacteriano obtenidas por edición genética (Montaguth, 2020).

Además, la región también fue precursora y hoy es protagonista en negocios de la bioeconomía relacionados con agricultura y ganadería baja en carbono, tales como las acciones nacionalmente apropiadas de mitigación (NAMA) en ganadería y café en Costa lica $(G I Z, 2019)$ y la ganadería sostenible en Brasil, Argentina, Uruguay y Colombia (FAO, 2019), así como en emprendimientos basados en el aprovechamiento productivo-comercial sostenible de la biodiversidad y de los servicios ecosistémicos y en la valorización y aprovechamiento de residuos en las cadenas del agro, entre muchos otros.

En todos los casos anteriores, los negocios de la bioeconomía han sido motores en el incremento de la competitividad del agro, la sostenibilidad ambiental y la generación de empleos e ingresos en los territorios rurales. Gracias a esto, el tema ha comenzado a tomar relevancia en la agenda pública y muchos tomadores de decisiones de la región se han convencido sobre su potencial y la necesidad y oportunidad de construir políticas, estrategias e iniciativas. De esta manera, no solo se impulsarán en mayor medida los negocios, sino que también se asegurará que estos se den en un marco de seguridad y sostenibilidad.

\section{LAS APUESTAS POLÍTICAS PARA INSTITUCIONALIZAR LA BIOECONOMÍA COMO ESTRATEGIA DE DESARROLLO: DESDE LAS INICIATIVAS ESPECÍFICAS A LAS ESTRATEGIAS DESTINADAS A LA BIOECONOMÍA}

Las experiencias de países de otras regiones del mundo evidencian que las dimensiones políticas e institucionales son determinantes en la transición hacia un modelo bioeconómico, puesto que se requiere liderazgo, articulación y gobernanza entre las instituciones para fomentar los desarrollos requeridos (Henry et al. 2014). Pese a lo anterior, en ALC la institucionalidad y los instrumentos de política pública relacionados con el fomento de la 
bioeconomía han estado relegados en comparación con el avance en los bionegocios. Solo recientemente, los tomadores de decisiones han reconocido el potencial de la bioeconomía, y los países han puesto en marcha instituciones y políticas dirigidas a su promoción.

Cada país de ALC ha establecido su propia agenda político-institucional en bioeconomía, basada en su disponibilidad y acceso a los recursos biológicos, capacidades tecnológicas, estructura productivo-comercial, objetivos de desarrollo nacional, entre otras. En este sentido, en el anexo 1 se resume la evolución de los marcos institucionales y de las políticas en diferentes senderos de la bioeconomía en la región. Se resalta que los sectores de mayor interés han sido los marcos regulatorios para el uso y aprovechamiento de la agrobiotecnología, la normativa en materia de biocombustibles, la gestión de la biodiversidad y el apoyo a los sistemas nacionales de CTI.

En lo referente a los sistemas nacionales de CTI, aunque la gran mayoría de los países cuenta con estrategias y planes nacionales y con programas sectoriales, lo cierto es que las limitaciones financieras experimentadas por la región han ocasionado que la inversión pública en investigación y desarrollo no solo sea baja e insuficiente, sino que también esté altamente concentrada en pocos países y enfocada en solventar problemas en rubros tradicionales (cultivos y ganadería) (IFPRI, 2020). A manera de ejemplo, mientras los países de altos ingresos invierten cerca del 2,81 USD en investigación y desarrollo por cada 100 USD de producción agrícola, en ALC únicamente Uruguay, Brasil, Chile y Argentina superan el monto de 1 USD.

Por otra parte, en las políticas asociadas a la bioeconomía en los sectores agroindustriales se presentan dos casos: los biocombustibles y la agrobiotecnología. El primero ha logrado materializar en instrumentos públicos estímulos para su producción, como beneficios tributarios y el establecimiento de zona francas (FAO, 2013), debido a la asociatividad gremial y a la capacidad técnica instalada del sector. En Brasil, por ejemplo, el desarrollo de toda la bioeconomía del país tuvo como punto de partida el Programa Nacional del Alcohol (Pro-Alcohol) y el resto de la institucionalidad desarrollada para fomentar el uso del etanol y el biodiésel como combustibles (RenovaBio y CBIO), la cual ha sobrepasado las fronteras y ha llevado los desarrollos 
tecnológicos a la industria de la agricultura, la química, la medicina, entre otras. En el caso de Argentina, desde la década de 1990 han existido incentivos a la agregación de valor, que hoy se materializan en el Régimen de Regulación y Promoción para la Producción y Uso Sustentables de Biocombustibles, enmarcado en las leyes 26.093 y 26.334 de 2006 (CEPAL et al. 2019). Ejemplos similares se pueden encontrar en Colombia y Paraguay, donde existen leyes y lineamientos para promover la producción sostenible de biocombustibles.

Para el caso de la agrobiotecnología, diez países de ALC cuentan con marcos nacionales de bioseguridad que regulan su uso. Uno de los pioneros fue Argentina, que en 1991 constituyó la Comisión Nacional de Bioseguridad (CONABIA) que posteriormente permitió un temprano aprovechamiento del potencial de estas tecnologías para el desarrollo productivo (CEPAL et al. 2019). Además de Argentina, países como Brasil, Chile, Colombia, Paraguay, Uruguay y Honduras, entre otros, han logrado consolidar a lo largo de los años la institucionalidad y las políticas para la generación y uso seguro y sostenible de las biotecnologías en la agricultura. Ahora, con las nuevas técnicas de mejoramiento genético, se espera que la regulación impulse la creación y el fortalecimiento de empresas de base biotecnológica que generen variedades propias de la región.

En cuanto a la valorización sostenible de la biodiversidad para nuevos negocios, es indispensable conocer en detalle y conservar la diversidad biológica (Hodson et al. 2019). Es por ello que todos los países de ALC han establecido al menos una política, plan o programa relacionado con la conservación de la biodiversidad. Sin embargo, aún existen grandes retos en materia de bioprospección y normatividad asociada al acceso a recursos genéticos, propiedad intelectual y beneficios para bionegocios que aprovechan esta riqueza natural.

Además de los avances en planes nacionales en CTI y normativas para los senderos de las bioenergías y biotecnologías o para el aprovechamiento de la biodiversidad, algunos países han progresado en la construcción de políticas orientadas exclusivamente a la bioeconomía, que promueven impulsar este abordaje como estrategia para la consecución de los objetivos de desarrollo 
sostenible de los países, territorios y cadenas. Dichos enfoques apuntan a construir un marco institucional que refleje el carácter transversal del tema. En este aspecto, se destacan los esfuerzos de Argentina, Costa Rica, Colombia y Uruguay:

- Argentina: fue uno de los primeros países de ALC que trabajó formalmente en la construcción de una hoja de ruta institucional para el desarrollo de su bioeconomía, por medio de la firma de convenios interministeriales y la instalación del Consejo Nacional de Bioeconomía liderado por el Ministerio de Ciencia, Tecnología e Innovación (Rodríguez, 2018) y la creación del Programa Nacional de Bioeconomía, como mecanismo específico para coordinar las actividades del MINAGRO en el tema. Además, en conjunto con la Bolsa de Cereales de Buenos Aires, se constituyó el Grupo Bioeconomía, como espacio para la identificación y promoción de las inversiones en las áreas vinculadas a la bioeconomía (CEPAL et al. 2019).

- Costa Rica: en 2020 se convirtió en el primer país de la región en oficializar una estrategia nacional en bioeconomía, la cual pretende apoyar los objetivos del país en materia de descarbonización y fomento sostenible de la competitividad a partir de la economía basada en el conocimiento y el aprovechamiento justo y equitativo de la biodiversidad. La estrategia cuenta con cinco ejes estratégicos: 1) bioeconomía para el desarrollo rural; 2) bioeconomía y desarrollo; 3) biorrefinerías de biomasa residual; 4) bioeconomía avanzada; y 5) bioeconomía urbana y ciudades verdes. Este proceso, que fue liderado por el Ministerio de Ciencia, Tecnología y Telecomunicaciones, se implementará en tres fases: de impulso (20202022); de escalamiento (2022-2026) y de consolidación (2026-2030) (MICITT, 2020).

- Colombia: en diciembre de 2020, el Ministerio de Ciencia, Tecnología e innovación lanzó la Misión de Bioeconomía, un plan que expresa y propone una estrategia nacional dedicada al desarrollo de la bioeconomía. Dicha misión cuenta con cinco áreas y desafios estratégicos: 1) Biodiversidad y sus servicios ecosistémicos; 2) Colombia 
Biointeligente; 3) Agro productivo y Sostenibe; 4) Biomasa y química verde y 5) Salud y Bienestar. La estrategia tiene dentro de sus metas que la bioeconomía aporte el $10 \%$ del PIB y genere 2.5 millones de empleos en el 2030 (MINCIENCIAS, 2020).

- Uruguay: está llevando a cabo el proceso de diseño de la estrategia nacional de bioeconomía sostenible. El proceso es liderado por la Oficina de Planeamiento y Presupuesto (que depende directamente de la Presidencia de la República) (FAO, 2018).

Finalmente, pese al avance de las iniciativas públicas en la región, aún falta concretar respaldos institucionales para la creación de instrumentos de financiación, planes de acción a largo plazo y sistemas de medición con indicadores adecuados para su monitoreo, seguimiento y evaluación. De igual forma, la coordinación de las intervenciones del sector público debe tener una visión integral de las distintas dimensiones de las políticas públicas, además de propiciar los esfuerzos del sector privado para la generación de empleo y la producción sostenible (Trigo et al. 2019).

\section{LOS ESFUERZOS PARA FOMENTAR LA COOPERACIÓN ENTRE LA REGIÓN Y CON EL RESTO DEL MUNDO}

Además de los esfuerzos que han realizado los sectores privado y público en la última mitad de la década, diversos organismos internacionales han impulsado la cooperación entre países y el intercambio de buenas prácticas, lecciones aprendidas y experiencias exitosas en la región. De la misma manera, han fomentado el trabajo colaborativo en la construcción de proyectos de investigación e inversión. Una de las primeras organizaciones regionales en apoyar el tema fue la Comisión Económica para América Latina y el Caribe (CEPAL), que capitalizó y continuó trabajando a partir de las redes y alianzas en los países después de la culminación de los proyectos ALC-UE.

Convencido del potencial del tema, en 2018 el Instituto Interamericano de Cooperación para la Agricultura (IICA) incluyó dentro de su Plan de Mediano 
Plazo para el período 2018-2022 el establecimiento del Programa Hemisférico de Bioeconomía y Desarrollo Productivo, que trabaja en los países de ALC en materia de: 1) generación de evidencia, sensibilización y formación de capacidades para los nuevos aprovechamientos de la bioeconomía en tomadores de decisión y actores del sector agro-rural; 2) formulación e implementación de herramientas y guías para construcción de hojas de ruta diferenciadas para el aprovechamiento de la bioeconomía de acuerdo a las potencialidades de los territorios y las cadenas de valor; 3) construcción de políticas, estrategias, normativas e instrumentos de mercados que viabilicen y posibiliten nuevos aprovechamientos productivos de la bioeconomía en el agro y lo rural; y 4) diseño e implementación de estrategias, planes, programas, proyectos e inversiones para fomentar nuevos modelos de negocios de la bioeconomía en los territorios rurales y las cadenas de valor de la agricultura (IICA, 2019b).

Además, junto a las representaciones del IICA en los países y diferentes socios y aliados, ese programa ha dirigido esfuerzos para apoyar el posicionamiento de la bioeconomía en los principales espacios técnicos y políticos de ALC, así como de la región en los escenarios mundiales de discusión de la bioeconomía. Concretamente, en octubre de 2019 la bioeconomía fue tema central en la reunión ministerial de la agricultura de las Américas y fue la base de una de las declaraciones ministeriales (IICA, 2019b). En octubre de 2020, el Instituto hospedó la Vigesimocuarta Conferencia Internacional de Bioeconomía Aplicada (ICABR), que estaba planeada para realizarse por primera vez en América Latina (Argentina), aunque debido a la pandemia de COVID-19 se llevó a cabo en modalidad virtual (IICA 2020b). En noviembre de 2020, ALC tuvo un inédito protagonismo en la Global Bioeconomy Summit, de la cual el IICA fue uno de los cinco socios oficiales (GBS, 2020).

Además del IICA y la CEPAL, otras organizaciones internacionales y regionales como la Organización Internacional del Trabajo (OIT), la Organización de las Naciones Unidas para la Educación, la Ciencia y la Cultura (UNESCO), el Banco Interamericano de Desarrollo (BID) y la Organización de las Naciones Unidas para la Alimentación y la Agricultura (FAO), han apoyado iniciativas tendientes a fomentar la cooperación regional en las áreas de políticas, indicadores, 
tecnología y formación de capacidades, entre otras. Como resultado de estos intereses y motivados por la invitación del gobierno de Argentina, en 2018 se realizó en Buenos Aires el Foro Latinoamericano de Bioeconomía. En este se constituyó la Red Latinoamérica de Bioeconomía, que tiene como objetivo Impulsar una estrategia de desarrollo regional que permita generar un intercambio de experiencias y proyectos colaborativos (MINCYT, 2019).

\section{APUNTES SOBRE LA AGENDA PENDIENTE}

ALC tiene una ventaja competitiva para convertir a la bioeconomía en el motor de la reactivación socioeconómica post COVID-19 y en una apuesta estratégica para la inserción exitosa en el nuevo entorno, dado que alberga a 8 de los 17 países más megadiversos del planeta, tiene más del $25 \%$ de las tierras cultivables y posee el $33.3 \%$ de los recursos de agua dulce del mundo, lo que la convierte en la región con el mayor potencial de generación de biomasa (CEPAL et al. 2019).

Sin embargo, para que la bioeconomía sea un modelo de desarrollo viable y aprovechable para los diferentes tipos de agricultura y territorios rurales de ALC y se generen encadenamientos con los restantes sectores de la economía, es indispensable que los desarrollos científico-tecnológicos estén acompañados de marcos normativos y políicos, así como de enfoques de mercado y mecanismos inclusivos que generen incentivos para que los agentes económicos de las cadenas de valor tomen la decisión de utilizar más eficientemente los recursos y procesos biológicos en sus modelos de producción, transformación y comercialización.

Entre las principales políticas habilitadoras de la bioeconomía se encuentran las siguientes: a) marcos regulatorios ambientales, sanitarios, agropecuarios y de salud que faciliten el fomento de la bioeconomía; b) instrumentos para fomentar la creación o el crecimiento de mercados de la bioeconomía (compras públicas, etiquetados, estándares, regulación y transparencia de mercados, etc.); c) estímulos económicos, financieros y fiscales (financiamiento, impuestos diferenciados, fondos de inversión, acompañamiento, etc.); d) generación y/o fortalecimiento de capacidades 
técnico-científicas para la innovación; e) políticas de localización industrial para la bioeconomía (promoción de clusters, capacitación, promoción de la inversión extranjera directa (IED), transferencia de tecnología, etc.); f) apoyo político al cambio social de base biológica (sensibilización sobre potencialidades); y g) fomento de la investigación, el desarrollo y la innovación (l+D+i) mediante programas de innovación, clusters, pilotos, generación de tecnologías, fortalecimiento de habilitantes, etc.).

Además de los esfuerzos que se puedan movilizar desde la región, para aprovechar la bioeconomía como una estrategia de desarrollo regional se requieren iniciativas supranacionales -lideradas por los países desarrollados-en materia de: i) un acuerdo más amplio sobre principios rectores para la formulación de políticas de bioeconomía global, ii) un marco de indicadores bioeconómicos creíbles y iii) una plataforma eficaz de gestión del conocimiento en bioeconomía (Chavarría et al. 2020).

\section{REFERENCIAS}

ARAGÃO, F. (2009). First transgenic geminivirus-resistant plant in the field (en línea). Nature Biotechnology, 27:1086-1088. doi: https://doi.org/10.1038/nbt1209-1086.

BISANG, R., CHAVARRÍA, H., \& TRIGO, E. (2019). ¿Cómo construimos la institucionalidad y las políticas públicas que se necesitan para desarrollar la bioeconomía en América Latina y el Caribe? San José, Costa Rica, IICA. Recuperado

de https://repositorio.iica.int/bitstream/handle/11324/8631/BVE20017763e.pdf? sequence $=1$ \&isAllowed $=y$.

BROOKES, G., \& BARFOOT, P. (2018). Environmental impacts of genetically modified (GM) crop use 1996-2016: Impacts on pesticide use and carbon emissions (en línea). GM Crops \& Food, 9(3):109-139. doi: 10.1080/21645698.2018.1476792.

CEPAL (COMISIÓN ECONÓMICA PARA AMÉRICA LATINA Y EL CARIBE, CHILE), FAO (ORGANIZACIÓN DE LAS NACIONES UNIDAS PARA LA ALIMENTACIÓN Y LA AGRICULTURA, ITALIA)., E IICA (INSTITUTO INTERAMERICANO DE COOPERACIÓN PARA LA AGRICULTURA, COSTA RICA). (2019). Perspectivas 
de la agricultura y del desarrollo rural en las Américas: una mirada hacia América Latina y el Caribe 2019-2020. San José, Costa Rica, IICA. Recuperado de https://repositorio.iica.int/bitstream/handle/11324/12380/BVE20107947e.pdf ? sequence $=1$ \&is Allowed $=$. .

CHAVARRÍA, H., TRIGO, E., VILLARREAL, F., \& ELVERDIN, P. (2020) BIOECONOMY: A SUSTAINABLE DEVELOPMENT STRATEGY (en línea). G20 2020. Recuperado de: https://www.g20-insights.org/policy briefs/bioeconomy-sustainabledevelopment-strategy/.

EUROPEAN COMMISSION. (2005). New perspectives on the knowledge based bioeconomy. Transforming life sciences knowledge into new, sustainable, eco efficient and competitive products. Bruselas, Bélgica.

EUROPEAN COMMISSION. (2008). From European fork to Latin American farm: an innovative networking platform for EU-LAC partnerships in food quality and safety R\&D (en línea). Bruselas, Bélgica. Recuperado de: https://cordis.europa.eu/project/id/7176/es.

EUROPEAN COMMISSION. (2013). Towards a Latin America \& Caribbean Knowledge Based Bio-Economy (KBBE) in Partnership with Europe (en línea). Bruselas, Bélgica. Recuperado de: https://cordis.europa.eu/project/id/264266/reporting/es.

EUROPEAN COMMISSION. (2017). Latin America, Caribbean and European Union Network on Research and Innovation (en línea). Bruselas, Bélgica. Recuperado de: https://cordis.europa.eu/project/id/31 1953/es.

FAO (ORGANIZACIÓN DE LAS NACIONES UNIDAS PARA LA ALIMENTACIÓN Y LA AGRICULTURA, ITALIA). (2013). La bioenergía en América Latina y el Caribe: el estado de arte en países seleccionados (en línea). Roma, Italia. Recuperado de: http://www.fao.org/3/a-as112s.pdf.

FAO (ORGANIZACIÓN DE LAS NACIONES UNIDAS PARA LA ALIMENTACIÓN Y LA AGRICULTURA, ITALIA). (2018). Uruguay rumbo a una estrategia nacional en bioeconomía (en línea). Roma, Italia. Recuperado de: http://www.fao.org/uruguay/noticias/detail/es/c/1103089/. 
FAO (ORGANIZACIÓN DE LAS NACIONES UNIDAS PARA LA ALIMENTACIÓN Y LA AGRICULTURA, ITALIA). (2019). Cambio climático y seguridad alimentaria y nutricional en América Latina y el Caribe (en línea). Santiago, Chile. 56 pp. Recuperado de: http://www.fao.org/3/ca2902es/CA2902ES.pdf.

GERMAN BIOECONOMY COUNCIL. (2014). Signpost in the Right Direction (en línea). Berlín, Alemania. Recuperado de: https://biooekonomierat.de/en/press/press-releases/press-release-boer140605/index.html.

GIZ (DEUTSCHE GESELLSCHAFT FÜR INTERNATIONALE ZUSAMMENARBEIT). (2019). NAMA Café de Costa Rica - Hacia un sector café bajo en emisiones len línea).

Recuperado

de: https://www.giz.de/en/downloads/giz2019 es Factsheet_NAMA\%20Cafe.p df.

GBS (GLOBAL BIOECONOMY SUMMIT). (2020). Official Partners of GBS2020 (en línea). Recuperado de: https://gbs2020.net/official-partnerships/.

HENRY, G., PAHUN, J., \& TRIGO, E. (2014). La bioeconomía en América Latina: oportunidades de desarrollo e implicaciones de política e investigación (en línea). FACES 42(4):(125-141). Recuperado de: http://nulan.mdp.edu.ar/2112/1/FACES n42-43 125-141.pdf.

HODSON, E. (2014). Hacia una bioeconomía en América Latina y el Caribe en asociación con Europa. Bogotá, Colombia, Pontificia Universidad Javeriana. HODSON, E., HENRY, G., \& TRIGO, E. (2019). La bioeconomía. nuevo marco para el crecimiento sostenible en América Latina (en línea). Bogotá, Colombia, Pontificia Universidad Javeriana. Recuperado de: http://repositorio2.iica.int/bitstream/handle/1 1324/8366/BVE190403022e.pdf ? sequence $=1$ \& is Allowed=y.

IFPRI (THE INTERNATIONAL FOOD POLICY RESEARCH INSTITUTE). (2020). Agricultural Science and Technology Indicators. Outputs for Latin America and the Caribbean (en línea). Recuperado de: https://www.asti.cgiar.org/lacoutputs.

IICA (INSTITUTO INTERAMERICANO DE COOPERACIÓN PARA LA AGRICULTURA, COSTA RICA). (2019a). Informe de la Conferencia de Ministros de Agricultura de las Américas y de la Vigésima Reunión Ordinaria de la Junta 
Interamericana de Agricultura (en línea). San José, Costa Rica. Recuperado de:

http://repositorio.iica.int/bitstream/handle/11324/8503/BVE20017732e.pdf.

IICA (INSTITUTO INTERAMERICANO DE COOPERACIÓN PARA LA AGRICULTURA, COSTA RICA). (2019b). Programa de bioeconomía y desarrollo productivo: abordajes conceptuales y metodológicos para la cooperación técnica (en línea). San José, Costa Rica. Recuperado de: https://repositorio.iica.int/handle/11324/7909.

IICA (INSTITUTO INTERAMERICANO DE COOPERACIÓN PARA LA AGRICULTURA, COSTA RICA). (2020a). Los biocombustibles líquidos en las Américas: situación actual y potencial de desarrollo (en línea). San José, Costa Rica. Recuperado de: http://biblioteca.iica.int/cgi-bin/koha/opacdetail.pl? biblionumber $=139983$.

IICA (INSTITUTO INTERAMERICANO DE COOPERACIÓN PARA LA AGRICULTURA, COSTA RICA). (2020b). Mayor encuentro global de bioeconomía reúne sector privado y representantes de universidades (en línea). San José, Costa Rica. Recuperado de: https://www.iica.int/es/prensa/noticias/mayorencuentro-global-de-bioeconomia-reune-sector-privado-y-representantesde.

ISAAA (INTERNATIONAL SERVICE FOR THE ACQUISITION OF AGRI-BIOTECH APPLICATIONS, ESTADOS UNIDOS DE AMÉRICA). (2018). Global Status of Commercialized Biotech/GM Crops in 2018: Biotech Crops Continue to Help Meet the Challenges of Increased Population and Climate Change. Ithaca, Nueva York, Estados Unidos de América. (ISAAA Brief No. 54).

JAMES, C. (1997). Global Status of Transgenic Crops in 1997 (en línea). Ithaca, Nueva York, Estados Unidos de América, ISAAA. Recuperado de: https://www.isaaa.org/resources/publications/briefs/05/download/isaadbrief-05-1997.pdf.

MICITT (MINISTERIO DE CIENCIA, TECNOLOGÍA Y TELECOMUNICACIONES, COSTA RICA). (2020). Estrategia Nacional de Bioeconomía Costa Rica 2020-2030 (en línea). San José, Costa Rica. Recuperado de: 
https://www.micit.go.cr/sites/default/files/estrategia_nacional bioeconomi a $\mathrm{Cr}$ corregido.pdf.

MINCIENCIAS (MINISTERIO DE CIENCIA, TECNOLOGÍA E INNOVACIÓN, COLOMBIA). (2020) Misión de Bioeconomía para una Colombia Potencia viva y diversa: Hacia una sociedad impulsada por el Conocimiento. Bogotá, Colombia.

MINCYT (MINISTERIO DE CIENCIA, TECNOLOGÍA E INNOVACIÓN, ARGENTINA). (2019). Primer Simposio Latinoamericano de Bioeconomía (en línea). Recuperado de: http://www.cursobioeconomia.mincyt.gob.ar/lersimposio-latinoamericano-de-bioeconomia/.

MONTAGUTH, S. (2020). Arroz editado genéticamente obtiene luz verde en EEUU y Colombia (en línea). Bogotá, Colombia, Agro-Bio. Recuperado de: https://www.agrobio.org/arroz-editado-geneticamente-obtiene-luz-verdeen-eeuu-y-colombia/.

OECD IORGANISATION FOR ECONOMIC CO-OPERATION AND DEVELOPMENT, FRANCIA). (2010). The bioeconomy to 2030: Designing a policy agenda (en línea). París, Francia. Recuperado de: https://www.oecd.org/futures/longtermtechnologicalsocietalchallenges/th ebioeconomyto2030designingapolicyagenda.htm.

REN21. (2019). Renewables 2019 Global Status Report (en línea). París, Francia. Recuperado de:

https://www.ren21.net/wpcontent/uploads/2019/05/gsr 2019 full report en.pdf.

RODRÍGUEZ, A. (2018). Bioeconomía en América Latina y el Caribe, 2018 (en línea). Santiago, Chile, Naciones Unidas. Recuperado de: https://repositorio.cepal.org/bitstream/handle/11362/44241/1/S1800922 es. pdf.

STOLF, R., OLIVEIRA, A. (2020). The Success of the Brazilian Alcohol Program (Proálcool) - A Decade-by-Decade Brief History of Ethanol in Brazil (en línea). Engenharia Agrícola, Jaboticabal, 40(2), 243-248. doi: http://dx.doi.org/10.1590/1809-4430-eng.agric.v40n2p243-248/2020.

THE WHITE HOUSE OFFICE. (2012). National Bioeconomy Blueprint (en línea). Washington D. C., Estados Unidos de América. Recuperado de: 
https://obamawhitehouse.archives.gov/sites/default/files/microsites/ostp/n ational bioeconomy blueprint april 2012.pdf.

TRIGO, E. (2016). Veinte años de cultivos genéticamente modificados en la agricultura argentina (en línea). Buenos Aires, Argentina, ArgenBio. Recuperado de: https://www.fiba.org.ar/wpcontent/uploads/2016/12/informe20gm.pdf.

TRIGO, E., \& HENRY, G. (2011). Una bioeconomía para América Latina y el Caribe: oportunidades y retos desde una perspectiva de políticas (en línea). Cali, Colombia, Oficina CIRAD ALCUE-KBBE, CIAT. Recuperado de: https://agritrop.cirad.fr/567664/1/document_567664.pdf.

TRIGO, E., CAP, E., MALACH, V., \& VILLARREAL, F. (2009a). Innovating in the Pampas: Zero-tillage soybean cultivation in Argentina (en línea). Spielman, DJ; Pandya-Lorch, R. In Millions Fed: Proven successes in agricultural development (pp. 59-64). Washington, D. C., Estados Unidos de América, IFPRI.

Recuperado

de: http://ebrary.ifpri.org/cdm/ref/collection/p15738coll2/id/130818.

TRIGO, E., CAP, E., MALACH, V., \& VILLARREAL, F. (2009b). The Case of Zero-Tillage Technology in Argentina (en línea). Washington, D. C., Estados Unidos de América, IFPRI. Recuperado de: https://www.ifpri.org/publication/casezero-tillage-technology-argentina.

TRIGO, E., REGÚNAGA, M., COSTA, R., \& COREMBERG, A. (2019). Bioeconomía en Argentina: alcances, situación actual y oportunidades para el desarrollo sustentable (en línea). In Hodson, E., Henry, G; Trigo, E. La bioeconomía. Nuevo marco para el crecimiento sostenible en América Latina (pp. 25-47). Bogotá, Colombia, Pontificia Universidad Javeriana. Recuperado de: http://repositorio2.iica.int/bitstream/handle/11324/8366/BVE190403022e.pdf ? sequence $=1$ \&isAllowed $=y$.

UNIDO (UNITED NATIONS INDUSTRIAL DEVELOPMENT ORGANIZATION). (2009). Report of UNIDO's Expert Group Meeting on Knowledge Based Bio Economy as Basis for Economic Development and Industrial Sustainability (en línea). Viena, Austria. Recuperado de: https://silo.tips/download/concepcionchile-november-30-december-2-2009. 
WALTZ, E. (2015). First stress-tolerant soybean gets go-ahead in Argentina (en línea). Nature Biotechnology, 33, 682. Recuperado de: https://doi.org/10.1038/nbt0715-682. 